\title{
Chiral symmetry breaking, color superconductivity and color neutral quark matter: a variational approach
}

\author{
Amruta Mishra ${ }^{1, \text { * }}$ and Hiranmaya Mishra ${ }^{2,}$, \\ 1 Institute für Theoretische Physik, Universität Frankfurt, D-60054 Frankfurt, Germany \\ ${ }^{2}$ Theory Division, Physical Research Laboratory, Navrangpura, Ahmedabad 380 009, India
}

(Dated: October 26, 2018)

\begin{abstract}
We investigate the vacuum realignment for chiral symmetry breaking and color superconductivity at finite density in Nambu-Jona-Lasinio model in a variational method. The treatment allows us to investigate simultaneous formation of condensates in quark antiquark as well as in diquark channels. The methodology involves an explicit construction of a variational ground state and minimisation of the thermodynamic potential. Color and electric charge neutrality conditions are imposed through introduction of appropriate chemical potentials. Color and flavor dependent condensate functions are determined through minimisation of the thermodynamic potential. The equation of state is calculated. Simultaneous existence of a mass gap and superconducting gap is seen in a small window of quark chemical potential within the model when charge neutrality conditions are not imposed. Enforcing color and electric charge neutrality conditions gives rise to existence of gapless superconducting modes depending upon the magnitude of the gap and the difference of the chemical potentials of the condensing quarks.

PACS numbers: 12.38.Mh, 24.85.+p
\end{abstract}

\section{INTRODUCTION}

The structure of vacuum in Quantum Chromodynamics (QCD) is one of the most interesting questions in strong interaction physics [1]. The evidence for quark and gluon condensates in vacuum is a reflection of its complex nature [2], whereas chiral symmetry breaking is an essential feature in the description of the low mass hadron properties. Due to the nonperturbative nature of QCD in this regime different effective models have been used to understand the nature of chiral symmetry breaking [3]. These have been constructed, for the most part, in the framework of a Nambu-Jona- Lasinio (NJL) model with a four fermion interaction.

Recently there has been a lot of interest in strongly interacting matter at high densities. In particular, a color superconducting phase for it involving diquark condensates has been considered with a gap of about $100 \mathrm{MeV}$. The studies have been done with an effective four fermion interaction between quarks [4], direct instanton approach 5] or a perturbative QCD calculation at finite density [6]. There has also been a study of this phase in NJL model 7]. Possibility of diquark condensates alongwith quark antiquark condensates has been considered in Ref. 8, 9, 10, 11, 12]. The natural place to look for such a phase seems to be in the interior of compact stellar objects like neutron star. However, to apply it to the case of neutron stars, the color and electric charge neutrality conditions need to be imposed for the bulk quark matter. Such an attempt has been made in Ref. 13] as well as in Ref. [14] where the lighter up and down quarks form two flavor color superconducting (2SC) matter while the strange quark do not participate in pairing. It has been shown, based upon comparison of free energy that a two flavor color superconducting phase would be absent in the core of neutron stars [13. Within NJL model in Ref. 14] it has been argued that such conclusions are consistent except for a small window in density range where superconducting phase is possible. There have also been studies to include the possibility of mixed phases [15] of superconducting matter demanding neutral matter on the average. In this context, there have been attempts to study the implications of vector interactions on the structure of the phase diagram 12 .

We had applied a different approach to study the problem in Ref. [9]. We considered a variational approach with an explicit assumption for the ground state having both quark antiquark and diquark condensates. The actual calculations are carried out for the NJL model such that the minimisation of the free energy density determines which condensate will exist at what density. In the present work we generalise the approach of Ref. [9] to include the conditions of color and electric charge neutrality. This leads to condensate functions which depend upon both color and flavor. Although, for simplicity, we shall be considering color superconductivity, this can be generalised to the

\footnotetext{
*Electronic address: mishra@th.physik.uni-frankfurt.de
}

${ }^{\dagger}$ Electronic address: hm@prl.ernet.in 
three flavor case to include color flavor locking. In fact here we shall also consider three flavors but having the $\mathrm{u}$ and $\mathrm{d}$ quarks taking part in diquark condensation. Although it might look rather complicated at the outset, the niceity of the approach is that within the model one can solve for the condensate functions explicitly which are flavor and color dependent.

We organize the paper as follows. In the next section we discuss the ansatz state with quark antiquark as well as diquark condensates. In section 3 we consider Nambu Jona-Lasinio model Hamiltonian and calculate the expectation value with respect to the ansatz state to compute the thermodynamic potential. We minimise the thermodynamic potential to calculate all the ansatz functions and the resulting mass as well as superconducting gap equations here. In section 4 we give the results and discuss them. Finally we summarise and conclude in section 5 .

\section{AN ANSATZ FOR THE GROUND STATE}

As noted earlier we shall include here the effects of both chiral symmetry breaking as well as diquark pairing. For the consideration of chiral symmetry breaking, we denote the perturbative vacuum state with chiral symmetry as $|0\rangle$. We shall then assume a specific vacuum realignment which breaks chiral symmetry because of interaction.

Let us note first the quark field operator expansion in momentum space given as [16, 17]

$$
\begin{aligned}
\psi(\mathbf{x}) & \equiv \frac{1}{(2 \pi)^{3 / 2}} \int \tilde{\psi}(\mathbf{k}) e^{i \mathbf{k} \cdot \mathbf{x}} d \mathbf{k} \\
& =\frac{1}{(2 \pi)^{3 / 2}} \int\left[U_{0}(\mathbf{k}) q_{I}^{0}(\mathbf{k})+V_{0}(-\mathbf{k}) \tilde{q}_{I}^{0}(-\mathbf{k})\right] e^{i \mathbf{k} \cdot \mathbf{x}} d \mathbf{k}
\end{aligned}
$$

where

$$
U_{0}(\mathbf{k})=\left(\begin{array}{c}
\cos \left(\frac{\phi^{0}}{2}\right) \\
\sigma \cdot \hat{k} \sin \left(\frac{\phi^{0}}{2}\right)
\end{array}\right), \quad V_{0}(-\mathbf{k})=\left(\begin{array}{c}
-\sigma \cdot \hat{k} \sin \left(\frac{\phi^{0}}{2}\right) \\
\cos \left(\frac{\phi^{0}}{2}\right)
\end{array}\right)
$$

The superscript 0 indicates that the operators $q_{I}^{0}$ and $\tilde{q}_{I}^{0}$ are two component ones which annihilate or create quanta acting upon the perturbative or the chiral vacuum $|0\rangle$. We have suppressed here the color and flavor indices of the quark field operators. The function $\phi^{0}(\mathbf{k})$ in the spinors in Eq.(2) are given as $\cot \phi_{i}^{0}=m_{i} /|\mathbf{k}|$, for free massive fermion fields, $i$ being the flavor index. For massless fields $\phi^{0}(|\mathbf{k}|)=\pi / 2$.

We now consider vacuum destabilisation leading to chiral symmetry breaking [9, 16, 17] described by,

$$
|v a c\rangle=\mathcal{U}_{Q}|0\rangle
$$

where

$$
\mathcal{U}_{Q}=\exp \left(\int q_{I}^{0 i}(\mathbf{k})^{\dagger}(\boldsymbol{\sigma} \cdot \mathbf{k}) h_{i}(\mathbf{k}) \tilde{q}_{I}^{0 i}(\mathbf{k}) d \mathbf{k}-\text { h.c. }\right) .
$$

In the above, $h_{i}(\mathbf{k})$ is a real function of $|\mathbf{k}|$ which describes vacuum realignment for quarks of a given flavor $i$. We shall take the condensate function $h(\mathbf{k})$ to be the same for $\mathrm{u}$ and $\mathrm{d}$ quarks and $h_{3}(\mathbf{k})$ as the chiral condensate function for the s-quark. Clearly, a nontrivial $h_{i}(\mathbf{k})$ shall break chiral symmetry. Sum over three colors and three flavors is understood in the exponent of $\mathcal{U}_{Q}$ in Eq.(4).

Having defined the state as in Eq.(3) for chiral symmetry breaking, we shall next define the state involving diquarks. We note that as per BCS result such a state will be dynamically favored if there is an attractive interaction between the quarks [18]. Such an interaction exists in QCD in the qq color antitriplet, Lorentz scalar and isospin singlet channel. In the flavor antisymmetric channel the interaction can be scalar, pseudoscalar or vector whereas in flavor symmetric channel only the axial vector channel is attractive. In the present work, we shall consider the ansatz state involving diquarks as

$$
|\Omega\rangle=\mathcal{U}_{d}|v a c\rangle=\exp \left(B_{d}^{\dagger}-B_{d}\right)|v a c\rangle
$$

where

$$
B_{d}^{\dagger}=\int\left[q_{r}^{i a}(\mathbf{k})^{\dagger} r f^{i a}(\mathbf{k}) q_{-r}^{j b}(-\mathbf{k})^{\dagger} \epsilon_{i j} \epsilon_{3 a b}+\tilde{q}_{r}^{i a}(\mathbf{k}) r f_{1}^{i a}(\mathbf{k}) \tilde{q}_{-r}^{j b}(-\mathbf{k}) \epsilon_{i j} \epsilon_{3 a b}\right] d \mathbf{k} .
$$

In the above, $i, j$ are flavor indices, $a, b$ are the color indices and $r(= \pm 1 / 2)$ is the spin index. As noted earlier we shall have $\mathrm{u}, \mathrm{d}(i=1,2)$ quark condensation. We have also introduced here (color, flavor dependent) functions $f^{i a}(\mathbf{k})$ 
and $f_{1}^{i a}(\mathbf{k})$ respectively for the diquark and diantiquark channels. As may be noted the state constructed in Eq. (5) is spin singlet and is antisymmetric in color and flavor. Clearly, by construction $f^{i a}(\mathbf{k})=f^{j b}(\mathbf{k})$ with $i \neq j$ and $a \neq b$. The corresponding Bogoliubov transformation for the operators is given by

$$
\left[\begin{array}{c}
q_{I r}^{i a \prime}(\mathbf{k}) \\
q_{I-r}^{k c l}(-\mathbf{k})^{\dagger}
\end{array}\right]=\left[\begin{array}{cc}
\cos f^{i a}(\mathbf{k}) & -2 r \epsilon_{i k} \epsilon_{3 a c} \sin f^{k c}(\mathbf{k}) \\
2 r \epsilon_{k i} \epsilon_{3 c a} \sin f^{i a}(\mathbf{k}) & \cos f^{k c}(\mathbf{k})
\end{array}\right]\left[\begin{array}{c}
q_{I r}^{i a}(\mathbf{k}) \\
\tilde{q}_{I-r}^{k c}(-\mathbf{k})
\end{array}\right]
$$

In a similar manner one can write down the Bogoliubov transformation for the antiquark operators corresponding to $|\Omega\rangle$ basis.

Finally, to include the effect of temperature and density we next write down the state at finite temperature and density $|\Omega(\beta, \mu)\rangle$ taking a thermal Bogoliubov transformation over the state $|\Omega\rangle$ using thermofield dynamics (TFD) as described in ref.s [19, 20]. We then have,

$$
|\Omega(\beta, \mu)\rangle=\mathcal{U}_{\beta, \mu}|\Omega\rangle=\mathcal{U}_{\beta, \mu} \mathcal{U}_{d} \mathcal{U}_{Q}|0\rangle
$$

where $\mathcal{U}_{\beta, \mu}$ is

$$
\mathcal{U}_{\beta, \mu}=e^{\mathcal{B}^{\dagger}(\beta, \mu)-\mathcal{B}(\beta, \mu)},
$$

with,

$$
\mathcal{B}^{\dagger}(\beta, \mu)=\int\left[q_{I}^{\prime}(\mathbf{k})^{\dagger} \theta_{-}(\mathbf{k}, \beta, \mu) \underline{q}_{I}^{\prime}(\mathbf{k})^{\dagger}+\tilde{q}_{I}^{\prime}(\mathbf{k}) \theta_{+}(\mathbf{k}, \beta, \mu) \underline{\tilde{q}}_{I}^{\prime}(\mathbf{k})\right] d \mathbf{k} .
$$

In Eq.(10) the ansatz functions $\theta_{ \pm}(\mathbf{k}, \beta, \mu)$ will be related to quark and antiquark distributions and the underlined operators are the operators in the extended Hilbert space associated with thermal doubling in TFD method. In Eq. (10) we have suppressed the color and flavor indices on the quarks as well as the functions $\theta(\mathbf{k}, \beta, \mu)$. Note that we have a proliferation of functions in the ansatz state $|\Omega, \beta, \mu\rangle$ - the (flavor dependent) chiral condensate function, the (color flavor dependent) quark as well as antiquark condensate functions and the (color flavor dependent) thermal functions. All these functions are to be obtained by minimising the thermodynamic potential. This will involve an assumption about the effective Hamiltonian. We shall carry out this minimisation in the next section.

\section{MINIMISATION OF THERMODYNAMIC POTENTIAL AND GAP EQUATIONS}

We shall work here in a Nambu-JonaLasinio model which is based on relativistic fermions interacting through local current- current couplings assuming that gluonic degrees of freedom can be frozen into point like effective interactions between the quarks. The Hamiltonian is given as

$$
\mathcal{H}=\sum_{i, a} \psi^{i a \dagger}\left(-i \boldsymbol{\alpha} \cdot \nabla+\gamma^{0} m_{i}\right) \psi^{i a}+\frac{g^{2}}{2} J_{\mu}^{a} J^{\mu a}
$$

Here $J_{\mu}^{a}=\bar{\psi} \gamma_{\mu} T^{a} \psi$ and, $m_{i}$ is the current quark mass which we shall take to be nonzero only for the case of strange quarks $(i=3)$. Of the two superscripts on the quark operators the first index ' $i$ ' refers to the flavor index and the second index, $a$ refers to the color index. The point interaction produces short distance singularities and to regulate the integrals we shall restrict the phase space inside the sphere $|\mathbf{p}|<\Lambda$ - the ultraviolet cutoff in the NJL model.

We next write down the expectation values of various operators in the variational ansatz state given in Eq. (8). Using the fact that the state in Eq. (8) arises from successive Bogoliubov transformations one can calculate these expectation values. These expressions would be used to calculate thermal expectation value of the Hamiltonian to compute the thermodynamic potential. With $\tilde{\psi}(\mathbf{k})$ as defined in Eq.(1), we evaluate the expectation values

$$
\left\langle\Omega(\beta, \mu)\left|\tilde{\psi}_{\alpha}^{i a}(\mathbf{k}) \tilde{\psi}_{\beta}^{j b}\left(\mathbf{k}^{\prime}\right)^{\dagger}\right| \Omega(\beta, \mu)\right\rangle=\delta^{i j} \delta^{a b} \Lambda_{+\alpha \beta}^{i a}(\mathbf{k}, \beta, \mu) \delta\left(\mathbf{k}-\mathbf{k}^{\prime}\right)
$$

and,

$$
\left\langle\Omega(\beta, \mu)\left|\tilde{\psi}_{\beta}(\mathbf{k})^{i a \dagger} \tilde{\psi}_{\alpha}^{j b}\left(\mathbf{k}^{\prime}\right)\right| \Omega(\beta, \mu)\right\rangle=\delta^{i j} \delta^{a b} \Lambda_{-\alpha \beta}^{i a, j b}(\mathbf{k}, \beta, \mu) \delta\left(\mathbf{k}-\mathbf{k}^{\prime}\right),
$$


where,

$$
\left.\Lambda_{ \pm}^{i a}(\mathbf{k}, \beta, \mu)=\frac{1}{2}\left[1 \pm\left(F_{1}^{i a}(\mathbf{k})-F^{i a}(\mathbf{k})\right) \pm\left(\gamma^{0} \cos \phi^{i}(\mathbf{k})\right)+\boldsymbol{\alpha} \cdot \hat{\mathbf{k}} \sin \phi^{i}(\mathbf{k})\right)\left(1-F^{i a}(\mathbf{k})-F_{1}^{i a}(\mathbf{k})\right)\right] .
$$

Here, the effect of diquark condensates and their temperature and/or density dependences are encoded in the functions $F^{i a}(\mathbf{k})$ and $F_{1}^{i a}(\mathbf{k})$ given as

$$
F^{i a}(\mathbf{k})=\sin ^{2} \theta_{-}^{i a}(\mathbf{k})+\sin ^{2} f^{i a}\left(C_{-}^{i a}(\mathbf{k})-\sin ^{2} \theta_{-}^{i a}(\mathbf{k})\right)\left(1-\delta^{a 3}\right),
$$

and,

$$
F_{1}^{i a}(\mathbf{k})=\sin ^{2} \theta_{+}^{i a}(\mathbf{k})+\sin ^{2} f_{1}^{i a}\left(\cos ^{2} \theta_{+}^{i a}(\mathbf{k})-S_{+}^{i a}(\mathbf{k})\right)\left(1-\delta^{a 3}\right) .
$$

Here, we have defined $C_{-}^{i a}=\left|\epsilon^{i i^{\prime}} \epsilon^{a a^{\prime}}\right| \cos ^{2} \theta_{-}^{i^{\prime} a^{\prime}}$ and $S_{+}^{i a}=\left|\epsilon^{i i^{\prime}} \epsilon^{a a^{\prime}}\right| \sin ^{2} \theta_{+}^{i^{\prime} a^{\prime}}$. The $\delta^{a 3}$ term indicates that the third color does not take part in diquark condensation. Further, we have introduced the notation $\phi_{i}(\mathbf{k})=\phi_{i}^{0}(\mathbf{k})-2 h_{i}(\mathbf{k})$. We also have

$$
\begin{aligned}
\left\langle\Omega(\beta, \mu)\left|\psi_{\alpha}^{i a}(\mathbf{x}) \psi_{\gamma}^{j b}(\mathbf{0})\right| \Omega(\beta, \mu)\right\rangle & =-\frac{1}{(2 \pi)^{3}} \int e^{i \mathbf{k} \cdot \mathbf{x}} \mathcal{P}_{+\gamma \alpha}^{i a, j b}(\mathbf{k}, \beta, \mu) d \mathbf{k}, \\
\left\langle\Omega(\beta, \mu)\left|\psi_{\alpha}^{i a \dagger}(\mathbf{x}) \psi_{\gamma}^{j b \dagger}(\mathbf{0})\right| \Omega(\beta, \mu)\right\rangle & =-\frac{1}{(2 \pi)^{3}} \int e^{i \mathbf{k} \cdot \mathbf{x}} \mathcal{P}_{-\alpha \gamma}^{i a, j b}(\mathbf{k}, \beta, \mu) d \mathbf{k},
\end{aligned}
$$

where,

$$
\begin{aligned}
\mathcal{P}_{+}^{i a, j b}(\mathbf{k}, \beta, \mu) & =\frac{\epsilon^{i j} \epsilon^{3 a b}}{4}\left[S^{i a, j b}(\mathbf{k}) \cos \left(\frac{\phi_{i}-\phi_{j}}{2}\right)\right. \\
& \left.+\left(\gamma^{0} \cos \left(\frac{\phi_{i}+\phi_{j}}{2}\right)-\boldsymbol{\alpha} \cdot \hat{\mathbf{k}} \sin \left(\frac{\phi_{i}+\phi_{j}}{2}\right)\right) A^{i a, j b}(\mathbf{k})\right] \gamma_{5} C
\end{aligned}
$$

and,

$$
\begin{aligned}
\mathcal{P}_{-}^{i a, j b}(\mathbf{k}, \beta, \mu) & =\frac{\epsilon^{i j} \epsilon^{3 a b} C \gamma_{5}}{4}\left[S^{i a, j b}(\mathbf{k}) \cos \left(\frac{\phi_{i}-\phi_{j}}{2}\right)\right. \\
& \left.+\left(\gamma^{0} \cos \left(\frac{\phi_{i}+\phi_{j}}{2}\right)-\boldsymbol{\alpha} \cdot \hat{\mathbf{k}} \sin \left(\frac{\phi_{i}+\phi_{j}}{2}\right)\right) A^{i a, j b}(\mathbf{k})\right] .
\end{aligned}
$$

Here, $C=i \gamma^{2} \gamma^{0}$ is the charge conjugation matrix (we use the notation of Bjorken and Drell) and the functions $S(\mathbf{k})$ and $A(\mathbf{k})$ are given as,

$$
S^{i j, a b}(\mathbf{k})=\sin 2 f^{i a}(\mathbf{k}) \cos 2 \theta_{-}^{i a, j b}(\mathbf{k}, \beta, \mu)+\sin 2 f_{1}^{i a}(\mathbf{k}) \cos 2 \theta_{+}^{i a, j b}(\mathbf{k}, \beta, \mu),
$$

and,

$$
A^{i j, a b}(\mathbf{k})=\sin 2 f^{i a}(\mathbf{k}) \cos 2 \theta_{-}^{i a, j b}(\mathbf{k}, \beta, \mu)-\sin 2 f_{1}^{i a}(\mathbf{k}) \cos 2 \theta_{+}^{i a, j b}(\mathbf{k}, \beta, \mu),
$$

In the above we have defined $\cos 2 \theta_{ \pm}^{i a, j b}=1-\sin ^{2} \theta_{ \pm}^{i a}-\sin ^{2} \theta_{ \pm}^{j b}$, with $i, j=1,2$ being the flavor indices and $a, b=1,2$ being the color indices and $i \neq j, a \neq b$.

Using Eq. (13) we have for the kinetic energy of the light quarks

$$
\begin{aligned}
T & \equiv\left\langle\Omega(\beta, \mu)\left|\psi^{\dagger}(-i \boldsymbol{\alpha} \cdot \boldsymbol{\nabla}) \psi\right| \Omega(\beta, \mu)\right\rangle \\
& =\frac{2}{(2 \pi)^{3}} \sum_{i=1,2, a=1,3} \int d \mathbf{k}|\mathbf{k}|\left(1-\cos 2 h_{i}(\mathbf{k})\left(1-F^{i a}-F_{1}^{i a}\right)\right),
\end{aligned}
$$

where, $F^{i a}$ and $F_{1}^{i a}$ are defined in equations (15) and Eq.(16). We have also subtracted the vacuum contributions.

Similarly the contribution from the interaction term in Eq.(11) after subtracting out the zero point perturbative energy turns out to be

$$
V \equiv\left\langle\Omega(\beta, \mu)\left|\frac{g^{2}}{2} J_{\mu}^{a} J^{\mu a}\right| \Omega(\beta, \mu)\right\rangle=V_{1}+V_{2}
$$


Here, the contribution $V_{1}$ arises from contracting $\psi$ with a $\psi^{\dagger}$ using Eq.s (12), (13) and is given as

$$
V_{1}=\frac{g^{2}}{2} \sum_{i=1,2}\left(\sum_{a=1,3} I_{v}^{i a^{2}}-2 \sum_{a=1,3} I_{s}^{i a^{2}}\right),
$$

with,

$$
I_{v}^{i a}=\frac{1}{(2 \pi)^{3}} \int d \mathbf{k}\left(F^{i a}-F_{1}^{i a}\right)
$$

and

$$
I_{s}^{i a}=\frac{1}{(2 \pi)^{3}} \int d \mathbf{k}\left(1-F^{i a}-F_{1}^{i a}\right) \sin 2 h_{i}(\mathbf{k}) .
$$

In this expression, we have neglected terms of the order $1 / N_{c}^{2}$ compared to unity. The term $V_{2}$ arises from contracting $\psi$ and a $\psi$ and $\psi^{\dagger}$ with another $\psi^{\dagger}$ using Eq. (18) and (19) and we have

$$
V_{2}=-\frac{4}{3} g^{2} I_{3}^{11,22} I_{3}^{12,21}
$$

with

$$
I_{3}^{i a, j b}=\frac{1}{(2 \pi)^{3}} \int d \mathbf{k} S^{i a, j b}(\mathbf{k}) \cos \left(\frac{\phi_{i}-\phi_{j}}{2}\right)
$$

where, $S^{i a, j b}$ has been defined in Eq.(20).

To calculate the thermodynamic potential we shall have to specify the chemical potentials relevant for the system. Here we shall be interested in the form of quark matter that might be present in compact stars older than few minutes so that chemical equilibriation under weak interaction is there. The relevant chemical potentials in this case then are the baryon chemical potential $\mu_{B}=3 \mu$, the chemical potential $\mu_{E}$ associated with electromagnetic charge $Q=\operatorname{diag}(2 / 3,-1 / 3,-1 / 3)$ in flavor space, and the two color electrostatic chemical potentials $\mu_{3}$ and $\mu_{8}$ corresponding to $U(1)_{3} \times U(1)_{8}$ subgroup of the color gauge symmetry generated by cartan subalgebra $Q_{3}=\operatorname{diag}(1 / 2,-1 / 2,0)$ and $Q_{8}=\operatorname{diag}(1 / 3,1 / 3,-2 / 3)$ in the color space. Thus the chemical potential is a diagonal matrix in color and flavor space, and is given by

$$
\mu_{i j, a b}=\left(\mu \delta_{i j}+Q_{i j} \mu_{E}\right) \delta_{a b}+\left(Q_{3 a b}+Q_{8 a b} \mu_{8}\right) \delta_{i j} .
$$

Here, $i, j$ are flavor indices and $a, b$ are color indices.

The total thermodynamic potential, including the contribution from the electrons, is then given by

$$
\Omega=T+V-\langle\mu N\rangle-\frac{1}{\beta} s+\Omega_{e}
$$

where, we have denoted

$$
\langle\mu N\rangle=\left\langle\psi^{i a \dagger} \mu_{i j, a b} \psi^{j b}\right\rangle=2 \sum_{i, a} \mu^{i a} I_{v}^{i a}
$$

with $\mu^{i a}$ being the chemical potential for the quark of flavor $i$ and color $a$, which can be expressed in terms of the chemical potentials $\mu, \mu_{E}, \mu_{3}$ and $\mu_{8}$ using Eq.(29). $\Omega_{e}=-\mu_{E}^{4} / 12 \pi^{2}$ is the electron free energy.

Finally, for the entropy density for the quarks we have [19]

$$
\begin{aligned}
s & =-\frac{2}{(2 \pi)^{3}} \sum_{i, a} \int d \mathbf{k}\left(\sin ^{2} \theta_{-}^{i a} \ln \sin ^{2} \theta_{-}^{i a}+\cos ^{2} \theta_{-}^{i a} \ln \cos ^{2} \theta_{-}^{i a}\right. \\
& \left.+\sin ^{2} \theta_{+}^{i a} \ln \sin ^{2} \theta_{+}^{i a}+\cos ^{2} \theta_{+}^{i a} \ln \cos ^{2} \theta_{+}^{i a}\right) .
\end{aligned}
$$

Now if we minimise the thermodynamic potential $\Omega$ with respect to $h_{i}(\mathbf{k})$, we get

$$
\tan 2 h_{i}(\mathbf{k})=\frac{\left(M_{i}-m_{i}\right) k}{\epsilon_{i}^{2}+g^{2} \sum_{a=1,3} I_{s}^{i a} m_{i}}
$$


where, $M_{i}=m_{i}+g^{2} \sum_{b} I_{s}^{i b}$ and $\epsilon_{i}=\sqrt{\left(\mathbf{k}^{2}+m_{i}^{2}\right)}$. Substituting this back in Eq. (26) we have the mass gap equation for the light quarks $\left(m_{i}=0\right)$

$$
M_{j}=g^{2} \sum_{a} I_{s}^{j a}=\frac{g^{2}}{(2 \pi)^{3}} \int \frac{M_{j}}{\sqrt{\mathbf{k}^{2}+M_{j}^{2}}} \sum_{a=1,3}\left(1-F^{j a}-F_{1}^{j a}\right) d \mathbf{k} .
$$

Clearly, the above includes the effect of diquark condensates as well as temperature and density through the functions $F$ and $F_{1}$ given in Eq.s (15) and (16) respectively.

Next, minimising the thermodynamic potential with respect to the diquark condensate functions leads, to

$$
\begin{aligned}
\tan 2 f^{12}(\mathbf{k}) & =\frac{\Delta_{12}}{\bar{\epsilon}-\bar{\nu}_{12}} \cos \left(\frac{\phi_{1}-\phi_{2}}{2}\right) \\
\tan 2 f^{11}(\mathbf{k}) & =\frac{\Delta_{11}}{\bar{\epsilon}-\bar{\nu}_{11}} \cos \left(\frac{\phi_{1}-\phi_{2}}{2}\right)
\end{aligned}
$$

In the above $\bar{\epsilon}=\left(\epsilon_{1}+\epsilon_{2}\right) / 2, \bar{\nu}_{11}=\left(\nu_{11}+\nu_{22}\right) / 2, \bar{\nu}_{12}=\left(\nu_{12}+\nu_{21}\right) / 2 . \nu^{i a}$ is the interacting chemical potential given as

$$
\nu^{i a}=\mu^{i a}-\frac{g^{2}}{4} \rho^{i}
$$

which may be expected in presence of a vectorial current-current interaction. In the above, $\rho^{i}=2 \sum_{a=1,3} I_{v}^{i a}$, with $I_{v}^{i a}$ as defined in equantion (25). Thus, it may be noted that the diquark condensate functions depend upon the average energy and the average chemical potential of the quarks that condense. We also note here that the diquark condensate functions depends upon the masses of the two quarks which condense through the function $\cos \left(\left(\phi_{1}-\phi_{2}\right) / 2\right)$ with $\cos \phi_{i}=\sin 2 h_{i}=M_{i} / \epsilon_{i}$, for u,d quarks which could be different when charge neutrality condition is imposed. Such a normalisation factor is always there when the condensing fermions have different masses as has been noted in Ref. 21] in the context of CFL phase.

In an identical manner the di-antiquark condensate functions are calculated to be

$$
\begin{aligned}
\tan 2 f_{1}^{12}(\mathbf{k}) & =\frac{\Delta_{12}}{\bar{\epsilon}+\bar{\nu}_{12}} \cos \left(\frac{\phi_{1}-\phi_{2}}{2}\right) \\
\tan 2 f_{1}^{11}(\mathbf{k}) & =\frac{\Delta_{11}}{\bar{\epsilon}+\bar{\nu}_{11}} \cos \left(\frac{\phi_{1}-\phi_{2}}{2}\right)
\end{aligned}
$$

Further, in Eq.s (35), (36), (38), (39), $\Delta_{12}=\left(2 g^{2} / 3\right) I_{3}^{11,22}, \Delta_{11}=\left(2 g^{2} / 3\right) I_{3}^{12,21}$ which satify the equations

$$
\begin{aligned}
\Delta_{12}=\frac{2 g^{2}}{3(2 \pi)^{3}} & \int d \mathbf{k}\left[\frac{\Delta_{11}}{\sqrt{\bar{\xi}_{-12}^{2}+\Delta_{11}^{2} \cos ^{2}\left(\frac{\phi_{1}-\phi_{2}}{2}\right)}}\left(\cos ^{2} \theta_{-}^{11}-\sin ^{2} \theta_{-}^{22}\right)\right. \\
& \left.+\frac{\Delta_{11}}{\sqrt{\bar{\xi}_{+12}^{2}+\Delta_{11}^{2} \cos ^{2}\left(\frac{\phi_{1}-\phi_{2}}{2}\right)}}\left(\cos ^{2} \theta_{+}^{11}-\sin ^{2} \theta_{+}^{22}\right)\right] \cos \left(\frac{\phi_{1}-\phi_{2}}{2}\right)
\end{aligned}
$$

and,

$$
\begin{aligned}
\Delta_{11}=\frac{2 g^{2}}{3(2 \pi)^{3}} & \int d \mathbf{k}\left[\frac{\Delta_{12}}{\sqrt{\bar{\xi}_{-11}^{2}+\Delta_{12}^{2} \cos ^{2}\left(\frac{\phi_{1}-\phi_{2}}{2}\right)}}\left(\cos ^{2} \theta_{-}^{11}-\sin ^{2} \theta_{-}^{22}\right)\right. \\
& \left.+\frac{\Delta_{12}}{\sqrt{\bar{\xi}_{+11}^{2}+\Delta_{12}^{2} \cos ^{2}\left(\frac{\phi_{1}-\phi_{2}}{2}\right)}}\left(\cos ^{2} \theta_{+}^{11}-\sin ^{2} \theta_{+}^{22}\right)\right] \cdot \cos \left(\frac{\phi_{1}-\phi_{2}}{2}\right)
\end{aligned}
$$

In the above, $\bar{\xi}_{ \pm i a}=\bar{\epsilon} \pm \bar{\nu}^{i a}$. Finally, minimisation of the thermodynamic potential with respect to the thermal functions $\theta_{ \pm}(\mathbf{k})$ gives

$$
\sin ^{2} \theta_{ \pm}^{i a}=\frac{1}{\exp \left(\beta \omega_{ \pm}^{i a}\right)+1}
$$


Various $\omega^{i a}$ s are given as follows.

$$
\begin{aligned}
& \omega_{-}^{11}=\omega_{-}+\delta_{\epsilon}-\delta_{\nu}^{11} \\
& \omega_{-}^{12}=\omega_{-}+\delta_{\epsilon}-\delta_{\nu}^{12} \\
& \omega_{-}^{21}=\omega_{-}-\delta_{\epsilon}+\delta_{\nu}^{12} \\
& \omega_{-}^{22}=\omega_{-}-\delta_{\epsilon}+\delta_{\nu}^{11} \\
& \omega_{+}^{11}=\omega_{+}+\delta_{\epsilon}+\delta_{\nu}^{11} \\
& \omega_{+}^{12}=\omega_{+}+\delta_{\epsilon}+\delta_{\nu}^{12} \\
& \omega_{+}^{21}=\omega_{+}-\delta_{\epsilon}-\delta_{\nu}^{11} \\
& \omega_{+}^{22}=\omega_{+}-\delta_{\epsilon}-\delta_{\nu}^{11}
\end{aligned}
$$

and, finally, for the noncondensing colors $\omega_{ \pm}^{i 3}=\epsilon^{i} \pm \nu^{i 3}$. As already mentioned, the first index refers to flavor and the second index refers to color. Here $\omega_{ \pm}=\sqrt{\Delta^{2} \cos ^{2}\left(\left(\phi_{1}-\phi_{2}\right) / 2\right)+\bar{\xi}_{ \pm}^{2}}, \delta_{\epsilon}=\left(\epsilon_{1}-\epsilon_{2}\right) / 2$ is half the energy difference of the two quarks which condense and e.g. $\delta_{\nu}^{11}=\left(\nu_{11}-\nu_{22}\right) / 2$, is half the difference of the chemical potentials of the two quarks which condense. Note that in the absence of imposing the charge neutrality condition all the four quasi particles will have the same energy $\omega_{-}$. Thus it is possible to have zero modes when charge neutrality condition is imposed depending upon the values of $\delta_{\epsilon}$ and $\delta_{\nu}$. So, although we shall have nonzero order parameter $\Delta$, there will be fermionic zero modes or the gapless superconducting phase [23, 24]. We shall discuss more about it section 4.

Next, let us focus our attention for the specific case of superconducting phase and the chemical potential associated with it. First let us note that the diquark condensate functions depend upon the average of the chemical potentials of the quarks that condense. Since this is independent of $\mu_{3}$ we can choose $\mu_{3}$ to be zero. In that case, $\mu^{11}=$ $\mu+2 / 3 \mu_{E}+\mu_{8} / \sqrt{3}=\mu^{12}$ and also $\mu^{21}=\mu-1 / 3 \mu_{E}+\mu_{8} / \sqrt{3}=\mu^{22}$. This also means that the average chemical potential of both the condensing quarks are the same and is equal to $\bar{\mu}=\mu+1 / 6 \mu_{E}+\mu_{8} / \sqrt{3}$. Further $\delta_{\nu}{ }^{12}=$ $\mu_{E} / 2-\left(g^{2} / 4\right)\left(\rho_{1}-\rho_{2}\right) / 2=\delta_{\nu}{ }^{11} \equiv \delta_{\nu}$. In such a situation, Eq. (40) and Eq. (41) are both identical and hence we shall have only one superconducting gap equation given as

$$
\Delta=\frac{2 g^{2}}{3(2 \pi)^{3}} \int d \mathbf{k}\left[\frac{\Delta}{\omega_{-}}\left(\cos ^{2} \theta_{-}^{12}-\sin ^{2} \theta_{-}^{21}\right)+\frac{\Delta}{\omega_{+}}\left(\cos ^{2} \theta_{+}^{12}-\sin ^{2} \theta_{+}^{21}\right)\right] \cos \left(\frac{\phi_{1}-\phi_{2}}{2}\right)
$$

With this condition,it is clear from Eq.s (43) that the quasi particle energies for each flavor becomes degenerate for both the colors which take part in condensation. Thus the quasi particle energies now become $\omega_{1}=\omega_{-}+\delta$ and $\omega_{2}=\omega_{-}-\delta$, for $\mathrm{u}$ and $\mathrm{d}$ quarks respectively, with $\delta=\delta_{\epsilon}-\delta_{\nu}$.

Now making the use of this dispersion relations and the mass gap equations Eq.(34) and the superconducting gap equation Eq.(44), the thermodynamic potential at zero temperature becomes, with $\delta=\delta_{\epsilon}-\delta_{\nu}$,

$$
\begin{aligned}
\Omega_{u, d} & =\frac{8}{(2 \pi)^{3}} \int d \mathbf{k}\left[|\mathbf{k}|-\frac{1}{2}\left(\omega_{-}+\omega_{+}\right)\right] \\
& +\frac{4}{(2 \pi)^{3}} \int d \mathbf{k}\left[\left(\omega_{-}+\delta\right) \theta\left(-\omega_{1}\right)+\left(\omega_{-}-\delta\right) \theta\left(-\omega_{2}\right)\right] \\
& +\frac{3 \Delta^{2}}{g^{2}}-\frac{g^{2}}{8} \sum_{i=1,2} \rho_{i}^{2}+\frac{1}{g^{2}}\left(M_{1}^{2}+M_{2}^{2}\right) \\
& -\sum_{i=1,2} \nu^{i 3} \rho^{i 3}+\frac{4}{(2 \pi)^{3}} \int d^{3} k\left[|\mathbf{k}|-\frac{\epsilon_{1}}{2} \theta\left(k-k_{f}^{13}\right)-\frac{\epsilon_{2}}{2} \theta\left(|\mathbf{k}|-k_{f}^{23}\right)\right] .
\end{aligned}
$$


The first three lines in Eq. (45) correspond to the contribution from the quarks taking part in the condensation while the last line is the contribution from the third color for the two light quarks.

In an identical manner one can calculate the thermodynamic potential for the strange quark sector with strange quarks and anti-quarks for the vacuum structure. The contribution to the thermodynamic potential then is given by

$$
\begin{aligned}
\Omega_{s} & =\frac{2}{(2 \pi)^{3}} \int d \mathbf{k} \sum_{a=1,3}\left[\sqrt{|\vec{k}|^{2}+m_{s}^{2}}-\sqrt{\mathbf{k}^{2}+M_{3}^{2}} \theta\left(|\mathbf{k}|-k_{f}^{3 a}\right)\right] \\
& +\frac{\left(M_{3}-m_{s}\right)^{2}}{g^{2}}-\frac{g^{2}}{8} \rho_{3}^{2}-\sum_{a} \nu^{3 a} \rho^{3 a} .
\end{aligned}
$$

Here, $\rho_{i}=\sum_{a=1,3} \rho^{i a}$ and $\rho^{i a}=2 I_{v}^{i a}$ for $i, a=1,2$, with $I_{v}^{i a}$ given in Eq. (25). For the third color, $\rho^{i 3}=\left(k_{f}^{i 3}\right)^{3} / 3 \pi^{2}$ and for the strange quark, $\rho^{3 a}=\left(k_{f}^{3 a}\right)^{3} / 3 \pi^{2}$. The fermi momenta are given by the usual relation $k_{f}^{i a}=\left(\nu_{i a}^{2}-M_{i}^{2}\right)^{1 / 2}$.

The total thermodynamic potential is given by including the contribution from the electrons and is given by

$$
\Omega=\Omega_{u, d}+\Omega_{s}-\frac{\mu_{E}^{4}}{12 \pi^{2}} .
$$

Thus the thermodynamic potential is a function of four parameters: the three mass gaps and a superconducting gap which needs to be minimised subjected to the conditions of electrical and color neutrality. The electric and charge neutrality constraints are given respectively as

$$
Q_{E}=\frac{2}{3} \rho_{1}-\frac{1}{3} \rho_{2}-\frac{1}{3} \rho_{3}-\rho_{e}=0
$$

and,

$$
Q_{8}=\frac{1}{\sqrt{3}} \sum_{i}\left(\rho^{i 1}+\rho^{i 2}-2 \rho^{i 3}\right)=0 .
$$

Eq.(47,49) and the superconducting gap equation Eq.(44) constitute the basis of the numerical calculations that we discuss below.

\section{RESULTS AND DISCUSSIONS}

For numerical calculations we have taken the values of the parameters of NJL model as follows: $g^{2} \Lambda^{2}=17.6$, $\Lambda=0.68 \mathrm{GeV}$ as typical values giving reasonable vacuum properties 10, 21, 22]. We might note here that the coupling $g^{2}$ introduced here is related to the usual scalar coupling of NJL model e.g. in Ref. [22] $G$ as $g^{2}=8 G$.

Current quark masses for $\mathrm{u}$ and $\mathrm{d}$ quarks are taken as zero and the current quark mass for strange quark is taken as $0.12 \mathrm{GeV}$. With this choice of parameters, the constituent quark masses at zero temperature and density are given as $M_{1}=0.35 \mathrm{GeV}=M_{2}$, and for strange quark $M_{3}=0.575 \mathrm{GeV}$.

In Fig [1 we have plotted the variation of masses with baryon density without diquark condensation and without imposition of the charge neutrality conditions. The decrease of masses with density is a reflection of the decrease of quark condensates with density.

Let us next discuss the case with diquark condensates along with quark antiquark condensates without imposing the charge neutrality condition. The numerical calculation for this case proceeds as follows. For a given chemical baryon potential, (and with $\mu_{3}=0=\mu_{8}$ ), the thermodynamic potential Eq. (47) is minimised with respect to the quark masses subjected to self consistently determining the interacting chemical potential using Eq. (37) and solving the superconducting gap equation Eq.(44). In Fig. 2 we have plotted the masses and the superconducting gap as a function of baryon chemical potential. The behaviour of masses are almost same to that without the diquark condensates. Here, however, we observe that for a small window in the quark chemical potential ( about $23 \mathrm{MeV}$ ), we have simultaneous existence of chiral symmetry breaking and color superconducting phase with the gap reaching a maximum upto $65 \mathrm{MeV}$ in this window. Including vector interactions similar conclusion of simultaneous existence of both the condenstes was noted earlier in Ref. [12]. However, in Ref. 12$]$ the vector interaction did not contribute to the superconducting gap equation nor to the mass gap equation unlike the case here. We have compared the pressure in both the cases in this regime and it appears that in this small window, existence of both the condensates has higher pressure than having only the quark antiquark condensates in the ground state. Beyond this window chiral symmetry is restored and the gap increases monotonically with chemical potential till the effect of cut off is felt and then it decreases. 


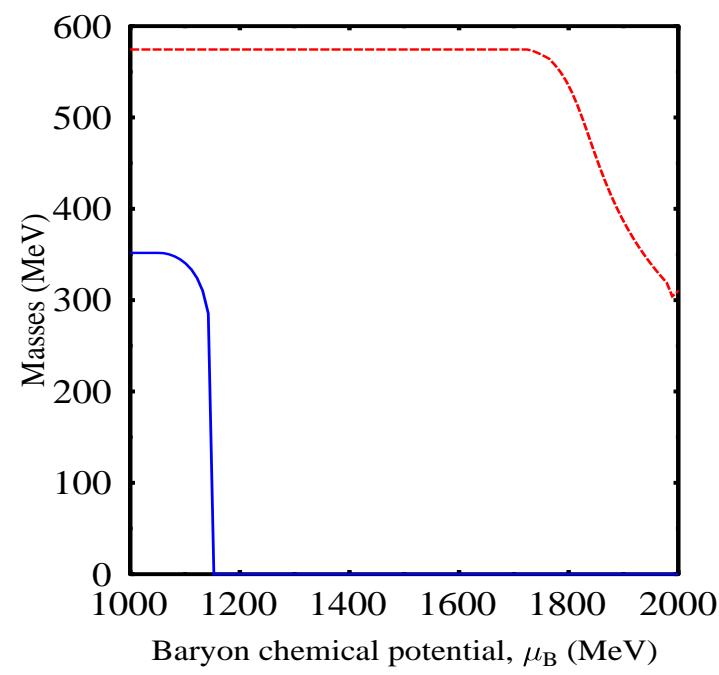

FIG. 1: Masses of the quarks as a function of baryon chemical potential. Solid curve refers to masses of $\mathrm{u}$ and $\mathrm{d}$ quarks while the dashed curve refers to strange quark mass.

We next discuss the results when charge neutrality conditions are imposed. As earlier, let us focus our attention first to the case without the diquark condensates. The results are shown in Fig. 3. Here, the d- quark mass vanishes earlier than that of $u$ quarks as the baryon chemical potential is increased. The reason is that to maintain electrical charge neutrality conditions the $d$ quark densities are almost twice that of $u$ quarks which makes the d quark antiquark condensates to vanish. In contrast to the rather sharp fall of the masses as compared to without imposition of electrical neutrality conditions, the u quarks remain massive much after d quark become massless (about $80 \mathrm{MeV}$ in the quark chemical potential window). The magnitude of electric chemical potential increases with densities till strange quarks begin to appear beyond which it starts decreasing so that charge neutrality conditions are maintained.

Next we show the results with diquark condensates with neutrality conditions. For a given quark chemical potential $\mu$, the interacting chemical potentials are determined using Eq.(37) with a trial value of $\mu_{E}$ and $\mu_{8}$. For high baryon chemical potential when chiral symmetry is restored for light quarks, the thermodynamic potential is varied with respect to the strange quark constituent mass after solving the superconducting gap equation Eq. (44). The values of $\mu_{E}$ and $\mu_{8}$ are varied so that the charge neutrality conditions Eq. (48) and Eq.(49) are satisfied. The results are shown in Fig.4. The behaviour of the superconducting gap is similar to that when charge neutrality condition are not imposed except that the magnitude of the gap decreases. The pressure of color and electrical neutral superconducting phase is lower than when neutrality conditions are not imposed as shown in Fig 5 Further, the pressure of color and electric neutral normal quark matter is always lower than that of the color and electrically neutral superconducting matter.

At high densities when chiral symmetry is restored for the light quarks, $\delta_{\epsilon}=0$ and $\delta=-\delta_{\nu}=-\left(\mu_{E} / 2-\left(g^{2} / 8\right)\left(\rho^{u}-\right.\right.$ $\left.\rho^{d}\right)$ ). For $\delta_{\nu}<0, \omega_{1}$, the quasi particle energy for u quarks is always positive and hence only the $\mathrm{d}$ quark contribution will be there in the second term of the thermodynamic potential given in Eq. (45). Further, it is easy to show that such a contribution arises when the magnitude of $\delta_{\nu}$, which is half the difference of the chemical potentials of the quarks which condense, is the same or greater than the superconducting gap, $\Delta$. When $\left|\delta_{\nu}\right|=\Delta$, the mode $\omega_{2}=\sqrt{\Delta^{2}+\left(\bar{\epsilon}^{2}-\bar{\nu}^{2}\right)}-\delta$, becomes gapless at the fermi sphere. For $\left|\delta_{\nu}\right|>\Delta$, the gapless modes occur at momenta higher than the (average) fermi momenta. In the present calculation this is the case for baryon chemical potential below $1600 \mathrm{MeV}$. The occurrence of such gapless superconducting modes in neutral quark matter was first emphasised in Ref. 25]. Because of this the number densities of $u$ and $d$ quarks participating in the superconducting phase are not the same in this region. This is plotted in Fig. [6. It may be noted that even in the gapless superconducting mode, the density of strange quarks is small but nonzero. 


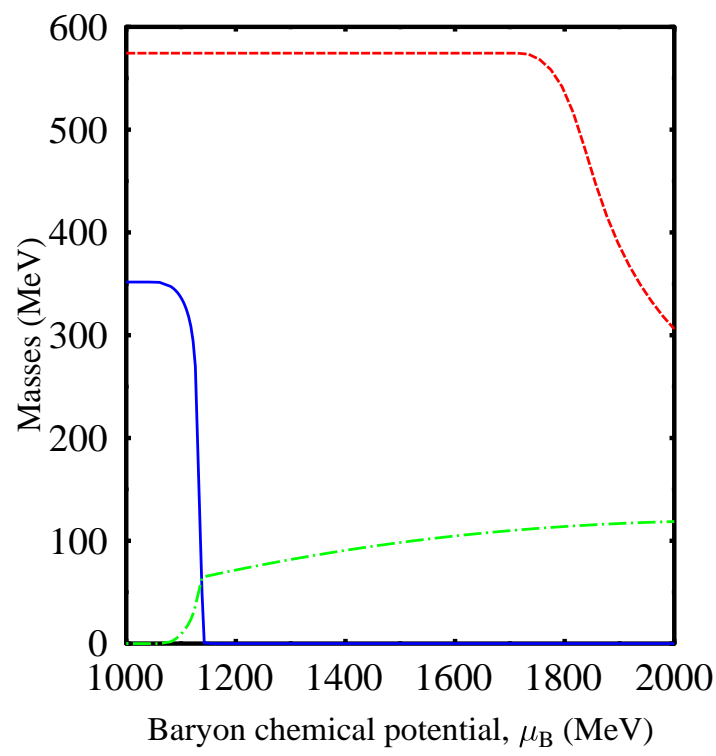

FIG. 2: Masses of the quarks and the superconducting gap as functions of baryon chemical potential for the case $\mu_{E}=0=\mu_{8}$. Solid, dashed and dot-dashed curves refer respectively to masses of up (down) quarks, strange quark and to the superconducting gap.

\section{SUMMARY}

We have analysed here in a current current point interaction model, the structure of vacuum in terms of quark antiquark as well as diquark pairs. The methodology uses an explicit variational construct of the trial state and is not based on a mean field calculations. Because of the point interaction structure we could solve for the gap functions explicitly. The distribution functions are also determined variationally. Because of the vector interaction, the chemical potentials are interaction dependent and are calculated self consistently. We find that there is a small window in baryon chemical potential (about $80 \mathrm{MeV}$ ) when both the condensates are nonzero.

To consider neutron star matter, we have imposed the condition of color and electric charge neutrality conditions through introduction of appropriate chemical potentials. It has been noted and emphasized earlier that projecting out the color singlet state from color neutral state costs negligible free energy for large enough chunk of color neutral matter [13].

The gap reduces when color and electric neutrality conditions are imposed. The pressure with the gap is always higher than free quarks when charge neutrality conditions are imposed. For slightly lower densities, but large enough to be in the chiral symmetry restored phase, there appears to be gapless modes available when the condensing quarks have a difference in chemical potentials which is larger than twice the superconducting gap. In all these calculations we have also included the effect of self consistently determined mass for the strange quark. In fact, in the gapless superconducting phase, the number densities of strange quarks is also nonzero.

We have focussed our attention here to the superconducting phase. The variational method adopted can be directly generalised to include color flavor locked phase and one can then make a free energy comparison regarding possibility of which phase would be thermodynamicaly favourable at what density. This will be particularly interesting for cooling of neutron stars with a CFL core. We have considered here homogeneous phase of matter. However, we can also consider mixed phases of matter with matter being neutral on the average. Some of these problems are being investigated and will be reported elsewhere [26]. 


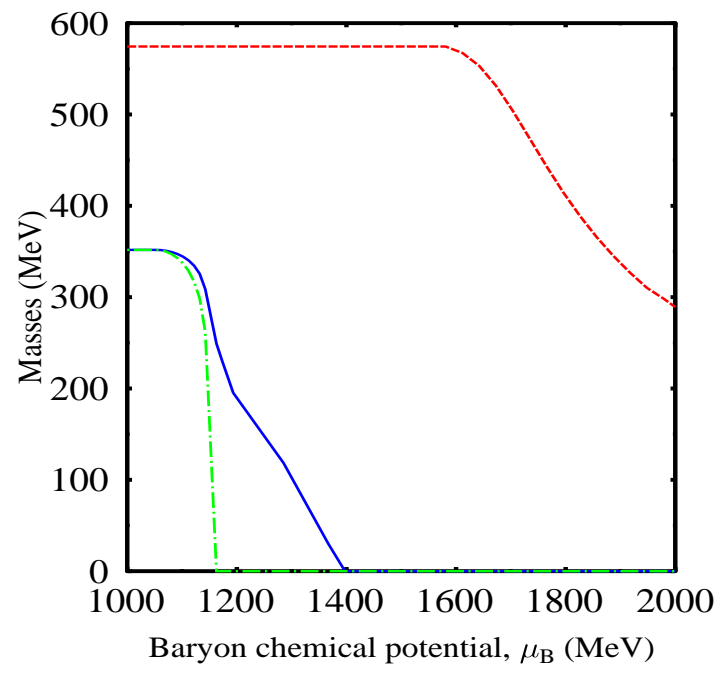

FIG. 3: Masses of the quarks without superconducting phase when electrical charge neutrality condition is imposed. Solid, dot-dashed and dashed curves refer respectively to masses of up, down and strange quark mass.

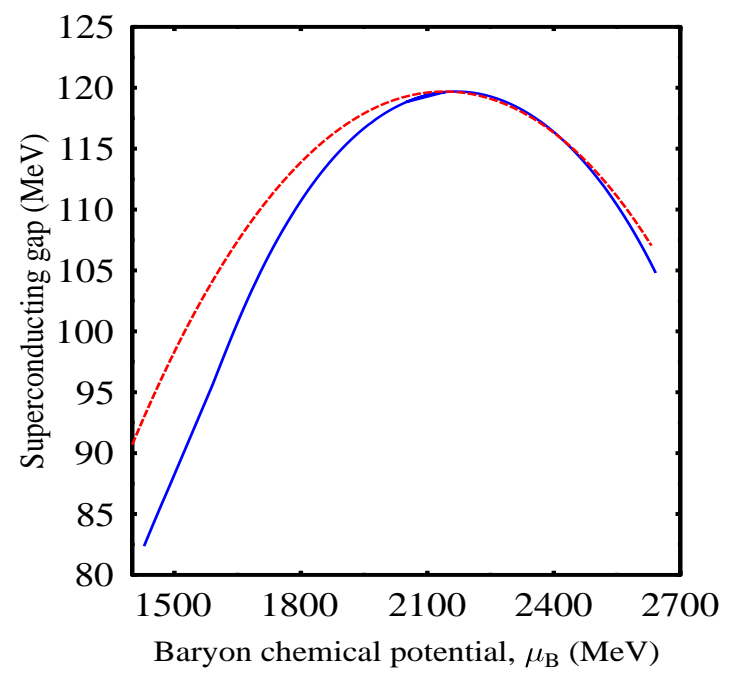

FIG. 4: Superconducting gap when color and electrical charge neutrality condition is imposed (solid curve). The dashed curve corresponds to when this condition is not imposed (i.e. $\left.\mu_{E}=0\right)$.

\section{Acknowledgments}

The authors would like to thank J.C. Parikh, D. H. Rischke, I. Shovkovy and M. Huang for many useful discussions. One of the authors (AM) would like to acknowledge financial support from Bundesministerium für Bildung und Forschung (BMBF) and Deutsches Elektronen Synchrotron (DESY), and Institut fuer Theoretische Physik, Frankfurt 


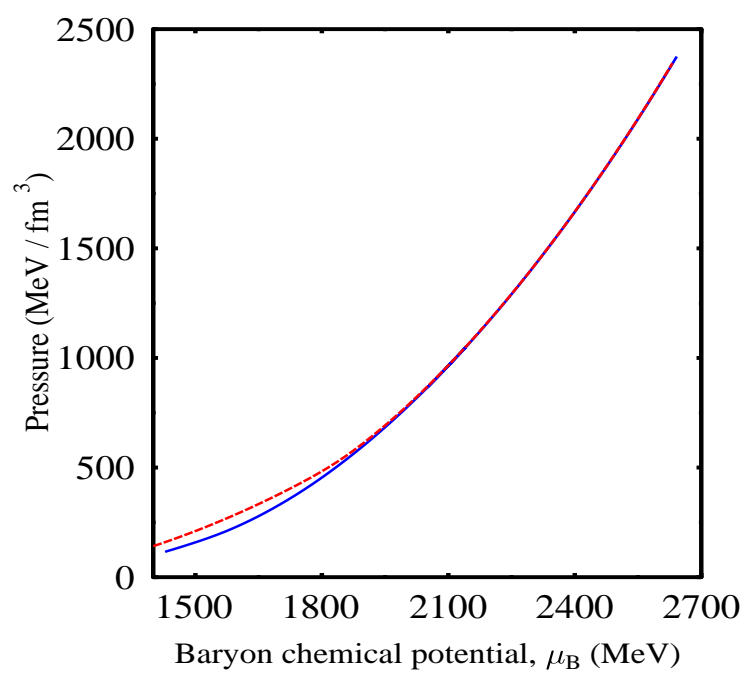

FIG. 5: Pressure as a function of baryon chemical potential when color and electrical charge neutrality condition is imposed (solid curve). The dashed curve corresponds to when this condition is not imposed.

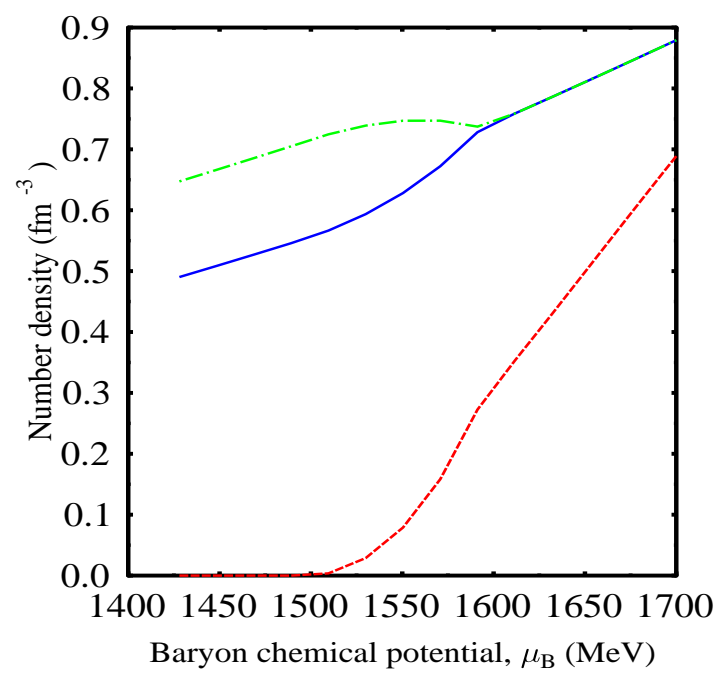

FIG. 6: Number densities of u quarks (solid) and d quarks (dot-dashed) participating in superconducting phase. The density of $s$ - quarks is also plotted (dashed curve).

University for warm hospitality.

[1] E.V. Shuryak, The QCD vacuum, hadrons and the superdense matter (World Scientific, Singapore, 1988).

[2] M.A. Shifman, A.I. Vainshtein and V.I. Zakharov, Nucl. Phys. B147, 385 (1979),ibid Nucl. Phys. B147, 448 (1979); ibidNucl. Phys. B147, 519 (1979). 
[3] Y. Nambu, Phys. Rev. Lett. 4, 380 (1960); A. Amer, A. Le Yaouanc, L. Oliver, O. Pene and J.C. Raynal,Phys. Rev. Lett. 50, 87 (1983a);ibid Phys. Rev. D 28, 1530 (1983); M.G. Mitchard, A.C. Davis and A.J. MAacfarlane, Nucl. Phys. B325, 470 (1989); B. Haeri and M.B. Haeri,Phys. Rev. D 43, 3732 (1991); V. Bernard,Phys. Rev. D 34, 1604 (1986); S. Schramm and W. Greiner, Int. J. Mod. Phys. E1, 73 (1992), J.R. Finger and J.E. Mandula, Nucl. Phys. B199, 168 (1982), S.L. Adler and A.C. Davis, Nucl. Phys.B244, 469 (1984), S.P. Klevensky, Rev. Mod. Phys.64, 649 (1992).

[4] M. Alford, K.Rajagopal, F. Wilczek, Phys. Lett. B 422, 247 (1998); ibidNucl. Phys. B537, 443 (1999).

[5] R.Rapp, T.Schaefer, E. Shuryak and M. Velkovsky, Phys. Rev. Lett. 81, 53 (1998);ibidAnn. Phys. 280, 35 (2000).

[6] D. Bailin and A. Love, Phys. Rep. 107, 325 (1984), D. Son, Phys. Rev. D 59, 094019 (1999); T. Schaefer and F. Wilczek, Phys. Rev. D 60, 114033 (1999); D. Rischke and R. Pisarski, Phys. Rev. D 61, 051501 (2000), D. K. Hong, V. A. Miransky, I. A. Shovkovy, L.C. Wiejewardhana, Phys. Rev. D 61, 056001 (2000).

[7] T.M. Schwartz, S.P. Klevansky, G. Papp, Phys. Rev. C 60, 055205 (1999).

[8] J. Berges, K. Rajagopal, Nucl. Phys. B538, 215 (1999).

[9] H. Mishra and J.C. Parikh, Nucl. Phys. A679, 597 (2001).

[10] M. Huang, P. Zhuang, W. Chao, Phys. Rev. D 65, 076012 (2002)

[11] D. Blaschke, M.K. Volkov and V.L. Yudichev, Eur. Phys. J. A 17, 103 (2003).

[12] M. Kitazawa, T. Koide, T. Kunihiro, Y. Nemeto, Prog. Theo. Phys. 108, 929 (2002).

[13] M. Alford and K. Rajagopal, JHEP 0206,031,(2002)

[14] A.W. Steiner, S. Reddy and M. Prakash, Phys. Rev. D 66, 094007 (2002).

[15] F. Neumann, M. Buballa and M. Oertel, Nucl. Phys. A714, 481 (2003).

[16] H. Mishra and S.P. Misra, Phys. Rev. D 48, 5376 (1993).

[17] A. Mishra and S.P. Misra, Z. Phys. C 58, 325 (1993).

[18] A.L. Fetter and J.D. Walecka, Quantum Theory of Many particle Systems (McGraw-Hill, New York, 1971).

[19] H. Umezawa, H. Matsumoto and M. Tachiki Thermofield dynamics and condensed states (North Holland, Amsterdam, $1982)$; P.A. Henning, Phys. Rep.253, 235 (1995).

[20] Amruta Mishra and Hiranmaya Mishra, J. Phys. G 23, 143 (1997).

[21] F. Gastineau, R. Nebauer and J. Aichelin, Phys. Rev. C 65, 045204 (2002).

[22] M. Buballa, M. Oertel, Nucl. Phys. A703, 770 (2002); P. Rehberg, S.P. Klevansky and J. Huefner, Phys. Rev. C 53, 410 (1996).

[23] A.A. Abrikosov, L.P. Gorkov, Zh. Eskp. Teor.39, 1781, 1960

[24] M.G. Alford, J. Berges and K. Rajagopal, Phys. Rev. Lett. 84, 598 (2000).

[25] Igor Shovkovy, Mei Huang, hep-ph/0302142 I. Shovkovy, M. Hanauske and M. Huang, Phys. Rev. D 67, 103004 (2003).

[26] A. Mishra and H.Mishra, in preparation 